\title{
Immunogenicity: prediction, detection and effective assay development
}

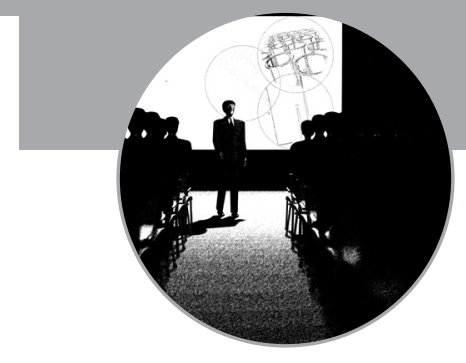

The Immunogenicity: Prediction, Detection and Effective Assay Development Conference

Munich, Germany, 21-23 June 2010

The Immunogenicity: Prediction, Detection and Effective Assay Development conference, organized by the Pharma iQ, a division of IQPC, was held in Munich, Germany, 2I-23 June of 20I0. The meeting covered a broad range of topics related to the unwanted immunogenicity of biotherapeutics. Perspectives from the US FDA and EMA were presented along with discussions focusing on analytical method development and validation, methodologies allowing for prediction of potential immunogenicity, risk-based assessment and mitigation of immunogenic potential of biologics, and other topics. The conference consisted of a preconference workshop and 2 days of plenary sessions, which included presentations as well as group discussions. Particular attention was drawn to the review of the FDA guidance and Committee for Medicinal Products for Human Use guidelines on immunogenicity evaluation. Presenters and meeting participants engaged in an active and productive discussion around scientific aspects involved in immunogenicity evaluation and methodologies involved during routine sample testing. Discussions also centered around challenges and opportunities related to detection and characterization of immunogenicity, as well as analysis of the latest technologies to enable accurate and meaningful interpretation of the results. The meeting attracted a broad range of participants from various sized companies, backgrounds and interests.

\section{Pre-conference workshop}

Harald Kropshofer, F Hoffmann La Roche, presented a workshop on technologies designed to assess immunogenicity. The interactive masterclass provided an opportunity to assess the antidrug antibody (ADA) prediction tools limitations, challenges and opportunities to positively impact assay development. During the workshop, participants reviewed ADA prediction tools and limitations; assays predictive for cytokine releaserelated infusion reactions; immunogenicity due to complement activation; and methods to predict anaphylaxis and anaphylactoid reactions.

Mechanistic background on immunogenicity was briefly described to underline importance of both T- and B-cell activation. Co-stimulatory molecules must be present in order to produce an active B-plasma cell (vs anergic B-cell). Co-stimulators, also called 'alarmins' or 'danger signals', include heat-shock proteins, Toll-like receptor proteins, endotoxins, proinflammatory cytokines and other molecules. As a consequence, some drugs may be more immunogenic under the conditions where pre-existing co-stimulatory signals are present (e.g., rheumatoid arthritis). Protein aggregates could also be viewed as an example of a danger signal [1].

Various prediction tools currently in use were presented, including screening for human leukocyte antigen (HLA) binding epitopes based on in silico analysis of protein sequence [2] and T-cell epitope validation by in vivo or in vitro screening protocols. Because HLA allotypes differ across various populations, certain allotypes may be enriched in particular disease conditions. HLA typing is therefore preferably performed in the target disease population. Currently, standards allowing for preclinical prediction tools have not been established, but are needed before the tools may be used in the routine evaluation of drug candidates. Immunogenicity prediction investigations are not currently considered a mandatory step by the regulatory organizations.

Several mitigation strategies were discussed. These include protein humanization and de-immunization based on modifying protein sequences to remove HLA binding epitopes. While having a high potential to reduce immunogenicity risk level, both methods have potential drawbacks, including the deletion of epitopes specific for regulatory T-cells, thereby increasing the risk for antibody generation. In all cases, new protein variants should be tested for efficacy and pharmacokinetic properties. It was suggested that prediction methodologies vary in terms of the end result, often overpredict and sometimes miss important epitopes. The area of B-cell epitope prediction was highlighted as a challenging but quickly developing area of interest.

\section{Boris Gorovits}

Pfizer, Andover, MA, USA

Tel.: + I 9782472292

Fax: + I 9782472842

E-mail: boris.gorovits@pfizer.com 
The presenter discussed infusion-related reactions, including drug-specific, IgE-related (Type 1, acute anaphylactic reactions), hypersensitivity reactions, cytokine-release syndrome and tumor lysis syndrome. Several factors could promote $\operatorname{IgE}$ induction, including drug type, T-cell epitope binding to class II HLA, low dose favoring Th2 induction, high solubility and stability, and others. Diagnostic biomarker tests include tryptase serum test, basophil activation test and skin-prick test. Hypersensitivity reactions are immune complex-mediated via drug-specific $\operatorname{IgG}(\operatorname{IgM})$ to complement interactions, and can be differentiated from Type I via a basophil activation test. In addition, complement activation-related pseudoallergy is driven by C3a and C5a complement factors, and does not involve specific immunoglobulin presence [3]. A porcine complement activation-related pseudoallergy test is often used as a predictive tool, but could be overpredictive.

The workshop concluded in a roundtable discussion focusing on various aspects of immunogenicity prediction and characterization.

\section{Plenary sessions}

- Immunogenicity prediction \& in vivo nonclinical evaluation

Harald Kropshofer reviewed immunogenicity prediction methodologies. T-cell-dependent responses represent approximately 85-95\% and T-cell-dependent responses and T-cellindependent responses are approximately $85-95 \%$ and $5-15 \%$ of the antibody formation, respectively. Dendritic cells (DCs) are key elements of the immune system and are present in various tissues, including blood. The DCs found in the blood (plasmacytoid DC) could be important in understanding T-cell-independent immunogenicity. The uptake of a drug protein by DCs is a key step in the T-cell-dependent induction of an anti-drug immunogenicity response. Inhibition of the uptake could prevent ADA formation. Migration of the drug protein to the B-cell-enriched locations (lymph nodes) is also required for successful ADA generation. T-cell independent induction is also driven by DCs, including plasmacytoid DCs. The proposed mechanism involves drug protein binding to the DCs that then deliver protein to the spleen where B-cells can be crosslinked and stimulated. The immunogenicity concept includes self versus foreign recognition by the T-cell receptors, but also involves the presence of danger signals (e.g., lipopolysaccharides and adjuvant) that trigger co-stimulation. These two events, self versus foreign recognition, plus the presence of a danger signal, determine whether the immune system will be stimulated. Therefore, it is proposed that co-stimulating molecules (danger signals) can be culprits in the process of breaking immune tolerance to otherwise self-proteins. Particular aggregates may stimulate T-cells by displaying newly formed epitopes, and therefore breaking the T- and/or B-cell tolerance. Aggregates may also function as danger signals, although receptors for such aggregates have not been reported [4]. Therefore two mechanisms of developing an aggregate with elevated immunogenicity were suggested: better uptake by DCs and/or T-cell independent induction of B-cells. The database of scientific facts allowing us to clearly link specific types of aggregates to the induction of anti-drug immune responses is still in its infancy. It is clear that several rather than one single condition is needed to induce immunogenicity during the administration of a biotherapeutic. The contribution of aggregates alone appears to be limited. Clinical data demonstrating a clear connection between levels of aggregated drug protein and increased incidences of ADAs are required to better understand the true role of protein aggregates in triggering immunogenicity.

Brigit Reipert, Baxter, reviewed mouse models designed for comparative immunogenicity studies during preclinical development of protein therapies. Models were designed to help develop improved versions of recombinant Factor VIII protein with the goal to increase protein halflife and decrease the levels of immunogenicity of the product. Next generations of Factor VIII are likely to be genetically modified and/or chemically modified protein molecules. Such modification could create neoantigens or generate inherent danger signals that could stimulate the innate immune system. Suitable animal models allowing for comparative analysis of immunogenicity potential for such products are expected to have the following properties:

- Mimic human disease;

- Express essential elements of the human immune system. Hyplotype HLA DRB1*1501, which is known to play an important role in human FVIII immunogenicity, was utilized in the described model;

- Induction of ADAs should depend on the same factors as in human patients. The mouse model presented does not express murine MHC class II molecules. Such 'humanized' 
hemophilic mice develop anti-FVIII antibodies that range in the isotype and neutralize the biological activity of FVIII;

- Differentiate between the native version of the drug and drug variants that are likely to carry neo-epitopes. For example, the ideal hemophilic animal model should not develop antiFactor VIII antibodies to the native factor VIII protein, but will be responsive to a modified Factor VIII by breaking immune tolerance.

The presenter described a murine Factor VIII knockout model capable of expressing the human Factor VIII protein. The model was utilized to compare several modified Factor VIII molecules with a clear difference in the immunogenicity potential. The model therefore allows drug candidates to be preselected and to exclude high immunogenicity risk protein constructs. The model may also help determine the role of aggregates in immunogenicity induction. Limitations of the model were discussed to include one of the human MHC class II haplotypes, since MHC protein is one of many proteins that modulate immunogenicity response in general. Additional evaluations in human trials will be required because the mouse model does not allow for complete prediction of the immunogenicity potential.

\section{- Review of regulatory guidelines for industry: ensuring compliance in assay development}

Jennifer Reed, US FDA, and Robin Thorpe, EMA, presented an overview of the FDA and Committee for Medicinal Products for Human Use (CHMP) guidance documents on the analysis of unwanted immunogenicity of biotherapeutics for industry. Potential consequences and incidences of immunogenicity responses vary widely across biological therapeutics. Both must be assessed in appropriate clinical studies. While the FDA document will address questions related to protein-based biotherapeutics, the new CHMP guidance is focusing on monoclonal antibody (mAb)-type biologics only. At the time of the conference, the FDA is in the process of collecting comments for the draft version of the guideline, while the CHMP document is under final review.

It is generally understood that the immunogenicity impact on patients is difficult to predict. Therefore, valid and sensitive assays for the detection of immunogenicity during clinical trials are essential for the development of all therapeutic protein products. Immunogenicity evaluation approaches should be based on potential risk to the patients where therapeutic proteins can be considered as high- to low-risk based on the ability to elicit immunogenicity responses with serious impact to the patients. Presenters stressed that factors that could influence immunogenicity of a biotherapeutic should be closely investigated and analyzed by the sponsor company. For example, chemical manufacturing control documentation should include a package with an analysis of the formulation, list and nature of excipients, impurities and so on. Reagents and assay methodologies should be in place to analyze ADA development on a proper time schedule (i.e., before the next study cohort). For lower risk molecules, assays are also expected, although banking of samples for retrospective analysis could be acceptable. When analyzing the risk level, information regarding the existence of similar products should be used to justify the approach taken. The presence of pre-existing antibodies could be considered as high risk. If pre-existing antibodies are found, the agency may request additional characterization of the responses. $\mathrm{mAb}$ biotherapeutics should not be viewed as a low-immunogenicity-risk class of therapeutics, and a case-by-case risk analysis should be conducted.

The CHMP document will address questions specific to immunogenicity of $\mathrm{mAb}$ drug compounds, including variability of immunogenicity and its consequences, particular problems experienced when developing screening and confirmatory assays, and strategies to be adopted when assessing neutralizing activity. Due to the multifaceted mechanism of action for a mAb drug molecule, tests for both the blocking of binding activity and interference with an immunobiological mode of action (e.g., blocking of effector function activity) should be considered when developing a neutralizing activity assay. Clinically relevant antibody levels should be defined based on the experience with a biotherapeutic molecule, therefore allowing the development and validation of assays that are fit for purpose. The guidance document describes several important assay elements: functionality (how is the target neutralization measured? how is the effector cell engagement measured?), sensitivity (what sensitivity is required to detect clinically relevant antibody levels?), and interference (what samples will be tested? what is the plan to mitigate matrix effects?). 
When developing positive control reagents, it was suggested that a purified version of antibody is preferred over a whole serum or a protein $G$ purified material, mainly due to the ease of calculating assay sensitivity. For a $\mathrm{mAb}$ molecule, a relevant specificity of the positive control is expected (e.g., with an anti-complementarity determining region [CDR] specificity). Specificity characterization should include whether ADAs can recognize the therapeutic protein or endogenous counterpart if any, or both; process/product contaminants; and any specific regions of the drug (e.g., Fc vs CDR for $\mathrm{mAbs})$. Characterization of the binding specificity may be requested based on the risk-level analysis of the molecule. Retrospective analysis may be acceptable, although reagents and methods should be in place.

Robustness evaluation should be suitable for the use of the assay and should help demonstrate which samples should be rejected based on storage and handling conditions. For example, for cell-based neutralizing antibody assays, cell passage number and medium components should be considered. Assay implementation will vary from product to product. An implementation plan should be available for review and feedback by the agency. The timing of sample collection should ensure the likelihood of detecting IgMand IgG-based responses, and to minimize interference from drug present in the assay matrix (i.e., sampling during washout period may be required). Early communication with regulatory agencies is important.

\section{- Current status of ADA assay methodologies}

Hishani Kirby, UCB Celltech, reviewed the current status of the immunogenicity analysis. Kirby presented a review of guidance documentation and several case studies. Immunogenicity testing is expected for biological therapeutics that are poorly defined, and presents a broad range of molecules. Existing guidelines aim to present a general approach that is common for all types of biological molecules. The immunogenicity risk level for a biotherapeutic is based on a component of frequency of ADA responses and severity of consequences. Both parameters vary across molecules. At this point, there are several white papers and guidelines with a common theme of a risk-based approach to immunogenicity assessment [5]. The presenter underlined that, when considering risk factors, one must consider factors affecting the potential incidence of the development of an ADA response. Risk assessment must be an integral part of clinical study design, including collection of appropriate samples (e.g., pre-treatment and sampling frequency) and existence of a washout period, as well as in some cases real-time bioanalysis for high-risk products. Interpretation of ADA data should be made in the context of pharmacokinetics/optical density (OD) readout and observed adverse toxicity effects. In presented case studies, the approach published by Ponce et al. was applied for both nonclinical and clinical immunogenicity evaluations [6]. The presenter suggested that ADA testing could be suspended if no immunogenicity development is observed in the study. Common parameters expected as part of characterization of ADA responses include detection of neutralizing activity, epitope mapping, isotyping and affinity evaluation. The need for characterization must clearly be value-driven and can be questioned in some cases. Various cut-point approaches for screening assays have been previously suggested [7]. The presenter argued that, in some cases, fixed and floating cut-points generate similar or the same reportable study results. Therefore, a floating cut-point could be substituted with a more straightforward, fixed cut-point approach. Similarly, the use of titers must be put into context because the use of exact titer values is unclear. Taken as a whole, the approach for immunogenicity evaluation has to be driven by the overall data set, including pharmacokinetics/pharmacodynamics information and toxicity, and should be value-driven and fit for purpose.

Darshana Jani, BiogenIdec, presented on evaluation of fluorometric and electrochemiluminescence (ECL) assay platforms for the detection of blocking antibodies in human serum. Dr. Jani reviewed and compared analytical platforms employed by Delfia and ECL technologies. A case study involving a soluble receptor IgG1 hinge Fc fusion protein was presented. Development of ADAs to the drug was initially tested using Delfia technology in a Phase I study involving healthy volunteers. Later, immunogenicity evaluation was conducted using ECL technology in a Phase II autoimmune population study. High ADA titers were observed in the Phase I clinical subjects. It was suggested that reactivity was associated with unidentified assay matrix effects. Inhibition experiments using a soluble version of the receptor and the drug target demonstrated that some, albeit not all, of the positive ADA reactivity was directed toward the drug target, and other factors contributed to the observed reactivity. Importantly, cell membrane 
preparation of the receptor drug target was used in the Delfia assay. Such preparations differed in the assay performance and had to be extensively requalified before use. Matrix interference observed in the Delfia assay platform was investigated by analyzing the effect of the rheumatoid factor and other autoimmune-related molecules, but a single definitive cause was not clearly identified. Later an ECL-based assay was employed in the Phase II study. ECL platforms utilized whole cells that express the receptor drug target molecule. The assay showed better reproducibility and lot-to-lot variability. In order to allow transition between assay platforms, blinded samples were generated and assay-to-assay conformance was confirmed.

Albert Torri, Regeneron Pharmaceuticals, spoke about a very important aspect of immunogenicity evaluation: automation of immunogenicity assays designed to improve analysis throughput and reproducibility. Torri discussed the considerable investment of money and people required for a successful introduction of automation. Benefits and drawbacks exist and are listed in Box $\mathbf{I}$.

Assay validation includes both a manual phase and automation-driven analysis. During the manual phase, additional tests are conducted to ensure the success of the automated assay platform. For example, additional testing includes extended reagent stability and incubation steps. System validation is included as part of the overall validation package. Automated assays generally produce consistent results, although assay failure rates may be higher versus manual. Warning systems must be set up to include warning lights, email, phone messaging, beepers and remote access. Throughput for the systems described in presentation can reach up to 2500 samples/month per system.

\section{- Three pillars of bioanalysis: immunogenicity, pharmacokinetics \& pharmacodynamics}

Andrew Warren, Novartis, presented an overview of various protein-protein interactions that may take place as a result of development of ADAs. Modeling of ADA effects should involve drug-ADA, drug-target and ADA-target interactions. These interactions can occur in vivo, but may also take place in vitro in the assay matrix while samples are tested. These interactions should be considered during the assay validation process. Pharmacokinetics/ pharmacodynamics modeling for mAb-based compounds should consider an elimination process for drug (free state), drug-target complex (bound state) and drug-ADA complexes. Importantly, modeling of an elimination process for the drug-target complex where target is a soluble versus a cell surface molecule predicts considerable difference in resulting pharmacokinetics profiles [8]. Assays designed to measure free-drug pharmacokinetics concentrations

\section{Box 1. Benefits and drawbacks to the introduction of automation.}

\section{Pros}

- Higher throughput, more analysis with fewer analysts

- Creates walk-away time

- Reduces assay variability due to reproducibility of performance

- Reduces risk for repetitive strain injuries

- Miniaturization: use of 384- and 1536-well plates is possible

\section{Cons}

- Expense

" Upfront, fully integrated system costs up to us $\$ 300,000$ or higher

- Operating cost is higher due to cost of disposables, increased reagent use, maintenance and calibration hardware

- Time to automate assays

- Need for highly trained specialists (both developing and maintaining skilled individuals)

- Space and facilities support

- Maintaining compliance requires continuous effort

- Single system error can potentially impact multiple plates/runs

- After system is developed, the laboratory is bound to the technology due to the substantial upfront investment

- Challenges include requesting or creating an interface between various software systems (this step often depends on vendor availability) 
generally involve capture or a detection step where drug molecule interaction with its target is utilized. Such assays are likely to be effected by the presence of drug neutralizing antibodies while binding antibodies may have little or no effect on results of a free-drug assay. To the contrary, in a total drug assay, capture and/or detection is often conducted using reagents binding to regions other than the $\mathrm{CDR}$ region of the $\mathrm{mAb}$ therapeutic. Therefore, the abilities of neutralizing and binding antibodies to alter results of total assay are reversed. Comparative analysis of pharmacokinetics and ADA results will therefore help to understand the type of ADA response developed. Similarly to the assays designed to detect drug concentrations, assays designed to detect drug targets can measure either free or bound molecules. Generally, ADA are not expected to have a considerable affect on the outcome of the drug target measurements.

The discussion was continued by Boris Gorovits, Pfizer. Immunogenicity evaluation is not generally the primary goal of a nonclinical toxicology study. The intent of immunogenicity evaluation greatly differs from that for the clinical studies. ADA evaluation is critical, but should be viewed as one of the components for interpreting effects observed during nonclinical evaluation, including alterations in pharmacokinetics, pharmacodynamics and toxicity end points. In general, nonclinical ADA studies cannot predict clinical immunogenicity, although there is some possibility of predicting the consequences of ADA development. During nonclinical evaluations, ADA data sets should be adjusted to the specific needs of each therapeutic development program [6]. ADA information should be incorporated together with the pharmacokinetics, pharmacodynamics, pathology and toxicity information to properly interpret nonclinical studies results. ADA information should be presented as part of the bioanalytical (BA) data package together with toxicokinetic information. To facilitate interpretation of nonclinical pharmacokinetics and ADA data for nonclinical studies, information should be assembled appropriately (e.g. based on individual and dosage groups, including baseline results) and presented in a summary table. Several case studies were presented to demonstrate potential value of the nonclinical evaluation of ADA development, including exposure (pharmacokinetics) effect and ability to explain and potentially predict toxicological observations.
Erik Foehr, BioMarin Pharmaceuticals, continued the discussion of the relationship between pharmacodynamics, pharmacokinetics and immunogenicity for biotherapeutics. These important elements should be considered in the development of any biologic, but particularly for high-immunogenicity-risk molecules. The idea was exemplified based on case studies of biologics developed at BioMarin. BioMarin research company is working on development of advanced treatment of metabolic disorders, including mucopolysaccharidosis and phenylketonuria. Enzyme-replacement therapeutics used to treat these conditions generally fall into the high immunogenicity risk category. The current strategy for immunogenicity evaluation, as described in the presentation, includes an analysis for total (binding) ADA, characterization of ADA isotype (IgG, $\operatorname{IgM}$ and $\operatorname{IgE}$ ) and detection of neutralizing ADA activities. For polyethylene glycol (PEG)-conjugated compounds, additional evaluation of anti-PEG IgG and anti-PEG IgM activities is included. Challenges related to the need for a specific IgM ADA-positive control were underlined.

A naglazyme case study was presented in detail. The drug mode of action includes cell uptake via interaction with mannose-6-phosphate receptor, trafficking to the lysosomal compartment and cleavage of the 4-O-sulfate on carbohydrate moiety. Therefore, two types of assays were developed to evaluate the presence of specific neutralizing activity; blocking cell surface binding and blocking specific enzymatic activity of the drug. It was shown that a relatively small fraction of ADA-positive samples contain cell surface blocking activity. An even smaller fraction of samples was able to exhibit enzyme-blocking activity. While positive seroconversion was observed in all patients treated, neutralizing activity could not be detected in the majority of the patient samples. Importantly, the presence of neutralizing activity did not translate into a significant pharmacodynamics effect. Lack of interference from neutralizing antibodies could be explained by low $\mathrm{pH}$ conditions of the lysosomal compartment where enzyme exhibits its activity.

Another case study involved a PEGylated version of phenylalanine hydroxylase $(\mathrm{PAH})$ utilized to treat phenylketonuria disorder. The company developed assays capable of detecting anti-PEG antibody to facilitate the interpretation of a potential effect on drug pharmacokinetics and safety profiles. Approximately $20-30 \%$ of drug-naive samples tested in the assay were 
demonstrated to have specific pre-existing antiPEG activity. All patients, including those that scored negative at the pretreatment, have developed anti-PEG antibodies following first administration of PEG-PAH. Similarly, all patients developed antiprotein moiety antibodies, but at a later time after multiple drug injections. In order to measure drug pharmacokinetics, samples were initially acidified, thereby opening specific epitopes on the protein moiety of the drug. The protein component was captured using a specific antiprotein drug-capture antibody reagent. Finally, detection was conducted using specific anti-PEG antibodies. Specificity of the method was tested to verify that PEG or unPEGylated PAH do not score in the assay.

\section{Summary}

The conference provided a broad scope of sessions regarding prediction, detection and mitigation of unwanted immunogenicity of biotherapeutics. Effective assay development and validation as well as sample testing were discussed. A review was presented from a global perspective, including US and European regulatory agencies. The meeting offered an exciting and engaging opportunity to be informed of the current status of the field and to keep abreast of the current regulatory views on the issue.

\section{Financial \& competing interests disclosure}

The author has no relevant affiliations or financial involvement with any organization or entity with a financial interest in or financial conflict with the subject matter or materials discussed in the manuscript. This includes employment, consultancies, honoraria, stock ownership or options, expert testimony, grants or patents received or pending, or royalties.

No writing assistance was utilized in the production of this manuscript.

\section{Bibliography}

1 Mahler HC, Friess W, Grauschopf U, Kiese S. Protein aggregation: pathways, induction factors and analysis. J. Pharm. Sci. 98(9), 2909-2934 (2009).

2 Koren E, De Groot AS, Jawa V et al. Clinical validation of the 'in silico' prediction of immunogenicity of a human recombinant therapeutic protein. Clin. Immunol. 124(1), 26-32 (2007).

3 Szebeni J. Complement activation-related pseudoallergy: a new class of drug-induced acute immune toxicity. Toxicology 216 (2-3), 106-121 (2005).
4 Braun A, Kwee L, Labow MA, Alsenz J. Protein aggregates seem to play a key role among the parameters influencing the antigenicity of interferon a in normal and transgenic mice. Pharm. Res. 14(10), 1472-1478 (1997).

5 Shankar G, Pendley C, Stein K. A risk-based bioanalytical strategy for the assessment of antibody immune responses against biological drugs. Nat. Biotechnol. 25 (5), 555-561(2007).

6 Ponce R, Abad L, Amaravadi L et al. Immunogenicity of biologically-derived therapeutics: assessment and interpretation of nonclinical safety studies Regul. Toxicol. Pharmacol. 54 (2), 164-182 (2009).
7 Shankar G, Devanarayan V, Amaravadi L et al. Recommendations for the validation of immunoassays used for detection of host antibodies against biotechnology products. J. Pharm. Biomed. Anal. 48, 1267-1281 (2008).

8 Joshi A, Bauer R, Kuebler P et al. An overview of the pharmacokinetics and pharmacodynamics of efalizumab: a monoclonal antibody approved for use in psoriasis. J. Clin. Pharmacol. 46, 10-20 (2006). 